\title{
«Sehen Sie mal, was ich sehe!»
}

Peter Berchtolda, Urs Zanoni ${ }^{\mathrm{b}}$

a PD Dr. med., b $\mathrm{MPH}, \mathrm{fmc}-$ Schweizer Forum für Integrierte Versorgung

\begin{abstract}
Die eindrucksvollen medizinisch-technischen Innovationen der letzten Jahre sind zum Wohl vieler Patienten. Gleichzeitig fragmentieren sie Behandlungswege und öffnen Qualitätslücken. Da gewinnt die Patientenperspektive eine besondere Bedeutung, denn es sind vor allem Patienten und Angehörige, die den gesamten Behandlungsprozess überblicken. Neue Forschungsarbeiten zeigen, wie eng Patientenzentrierung, Integrierte Versorgung und Qualität zusammenhängen.
\end{abstract}

"At a time when anger and distrust seem ubiquitous in the health systems of so many countries, asking patients to report on the quality of their care may bring clinicians and those they serve closer together.» (Prof. Dr. Thomas Delbanco)

\section{Hintergrund}

Das Zitat des Harvard-Professors Thomas Delbanco, 1996 im British Medical Journal publiziert, könnte nicht aktueller sein: Soll die Behandlung wirklich patientenzentriert sein, ist die Versorgung viel stärker als bisher durch die Augen der Patienten zu sehen und zu planen [1]*. Denn heute stehen viel mehr Behandlungsoptionen zur Wahl, in erster Linie als Folge der medizinischen und medizinisch-technischen Innovationen der letzten Jahre: einerseits zum Vorteil der Patienten, weil heute mehr Krankheiten erfolgreich behandelt und mehr Leiden wirksam gemildert werden können; andererseits zum Nachteil der Patienten, weil sich dieses Mehr-Wissen und MehrKönnen zwangsläufig auf mehr Fachpersonen und Institutionen verteilt, was den Behandlungsweg eines einzelnen Patienten ebenso zwangsläufig in immer kleinere Abschnitte zergliedert und Qualitätslücken öffnet.

Für die Patienten bedeutet diese Entwicklung eine wachsende Zahl an mitverantwortlichen Fach- und Ansprechpersonen. Ausserdem sind die Rollen und Aufgaben vor allem von spezialisierten Fachpersonen für die Patienten viel weniger identifizierbar als früher.

Dies distanziert die Betreuenden bzw. Behandelnden zunehmend von ihren Patienten; diese wiederum fühlen sich immer öfters orientierungslos und im Wirrwarr der Informationen verloren. Es erstaunt kaum, dass wir diese Wahrnehmung besonders häufig bei chronisch bzw. mehrfach kranken Patienten mit langem Betreuungsverlauf finden [2].

\section{Sicht der Patienten ...}

Vor diesem Hintergrund gewinnt das Eingangszitat von Thomas Delbanco eine weitergehende Bedeutung: Es sind vor allem oder sogar ausschliesslich die $\mathrm{Pa}$ tienten (und allenfalls ihre Angehörigen), die über Erfahrungen aus erster Hand mit allen einzelnen Fragmenten ihrer Behandlung bzw. den verschiedenen Fachteams verfügen und damit über den gesamten Behandlungsprozess. Keine anderen Instanzen, auch nicht Hausärzte oder Case Manager, können diese Erfahrung ersetzen. Hausärzte und Spitex-Pflegende spielen zwar eine zunehmend bedeutende Rolle bei der Koordination der Behandlung und Betreuung ihrer Patienten. Gleichzeitig ist ihr Beitrag aus Sicht der Betroffenen ebenfalls «nur» ein (wenn auch grosses und wiederkehrendes) Fragment im gesamten Ablauf.

Damit wird deutlich, dass die Sicht des Patienten, der Patientin zu einer essentiellen Quelle wird, wenn es um die Beobachtung und Optimierung der Behandlungsprozesse und deren Qualität geht. Während Fachpersonen, gleich welcher Berufsgruppe, zwangsläufig ein einzelnes Behandlungsfragment und häufig ein einzelnes Organ im Blick haben, erleben die Patienten und ihre Angehörigen (oder andere Vertrauenspersonen) immer den gesamten Betreuungsprozess und den ganzen Menschen.

Solche Erkenntnisse haben in den letzten Jahren zahlreiche Initiativen und Projekte mit dem Ziel ausgelöst, Patientenperspektive und Behandlungen enger $\mathrm{zu}$ verzahnen und so eine wirklich patientenzentrierte Versorgung zu fördern. Zum Beispiel an der Universität Heidelberg, wo erstmals ein Fragebogen entwickelt und validiert wurde, mit dem Qualitätslücken über ganze Behandlungsabläufe aus Patientensicht identifiziert werden können [3]. Oder im englischen Department of Health, welches das Strategiepapier «No deci- 
sion about me, without me» veröffentlichte mit der Absicht, Patienten im Sinne des Shared decision making direkter in ihre Behandlung einzubinden [4].

\section{... und Integrierte Versorgung}

Daher erstaunt es nicht, dass die Betroffenen rasch auf Koordination, Vernetzung und Kontinuität der Behandlungsabläufe zu sprechen kommen, wenn sie zu patientenzentrierter Behandlung befragt werden. Patienten wissen sehr wohl, was (bessere) Vernetzung und Koordination für ihre Behandlung bedeutet - und vor allem spüren sie rasch, wenn es fehlt [5]. Neue

Patienten wissen sehr wohl, was Vernetzung und Koordination für ihre Behandlung bedeutet und spüren rasch, wenn es fehlt.

Forschungsarbeiten zeigen denn auch, wie eng Patientenzentrierung und vernetzte Behandlung (Integrierte Versorgung) zusammenhängen [6]. Mit gutem Grund: Für einen Patienten mit seiner ganzen Krankheitsgeschichte und dem ganzen Behandlungsplan vor Augen sind Fragmentierungen und Unterbrechungen zum einen sichtbar, zum anderen ein Problem. Darum ist es für Patienten fast selbstverständlich, dass allfällige Qualitätslücken vor allem an den Schnittlinien auftreten [7].

\section{Patientenzentrierung konkret}

Was also heisst Patientenzentrierung im medizinischen und pflegerischen Alltag? Und wie können Patienten stärker in die Entscheidfindung entlang der gesamten Behandlungspfade einbezogen werden? $\mathrm{Zu}$ diesen Fragen wurde in der Vergangenheit bereits sehr viel diskutiert und publiziert, so viel, dass $\mathrm{Pa}$ tientenzentrierung oft mehr Kopfschütteln denn Interesse findet und eher verulkt denn ernsthaft diskutiert wird. "Der Patient steht im Zentrum und darum im Weg», ist ein bekanntes Beispiel dafür. Kein Wunder, denn die meisten dieser Diskussionen werden über, aber kaum je mit Patienten geführt.
Aufgrund neuerer Forschungsresultate lässt sich der abstrakte Begriff «Patientenzentrierung» in vier Dimensionen operationalisieren:

1. Verständliche Information der Patienten, d.h. Patienten und Angehörige erhalten ausreichende Kenntnisse und Orientierung zum gesamten Behandlungsprozess (und nicht nur über einzelne Episoden), so dass sie sich über Ablauf, Outcome und Risiken ein Bild machen können;

2. Einbezug der Patienten in die Entscheidfindung, d.h. Patienten geben nicht nur ihre Zustimmung zu einer Behandlungsempfehlung (im Sinne des «informed consent»), sondern kennen allfällige Behandlungsalternativen, und/oder es wird ihnen von den Behandelnden eine Zweitmeinung empfohlen;

3. Koordination und Kontinuität der Behandlung und Betreuung, d.h., die betreuenden Fachpersonen bewerten Untersuchungsbefunde einheitlich und ziehen daraus zuhanden der Patienten und Angehörigen übereinstimmende Schlüsse zu Diagnose, Behandlung und Prognose;

4. Kohärente Kommunikation zwischen den Leistungserbringenden, d.h., die betreuenden Fachpersonen kennen die Rollen und Funktionen der jeweils anderen, gewährleisten gemeinsam eine optimale Betreuung und respektieren insbesondere direkte (und koordinierende) Ansprechpersonen der Patienten und ihrer Angehörigen.

Diese Forschungsresultate illustrieren eindrucksvoll, was aus Patientensicht relevant ist und wie Qualität in all ihren Facetten von einer funktionierenden Verzahnung aller beteiligten Institutionen und Fachpersonen abhängig ist. Die Sicht der Patienten einnehmen heisst nicht, die Meinung der Patienten zu übernehmen. "Sehen Sie mal, was ich sehe!», ist vielmehr eine Einladung an Fachpersonen, die Gesamtsicht der Patienten bei der Planung der einzelnen Behandlungsund Betreuungsfragmente zu berücksichtigen. Und gleichzeitig die Chance, überzogene oder unrealistische Ansprüche der Betroffenen anzusprechen.
Korrespondenz:

PD Dr. med. Peter Berchtold Geschäftsstelle

Forum Managed Care Zugerstrasse 193

CH-6314 Neuägeri

peter.berchtold[at]fmc.ch

\section{Nationales Symposium Integrierte Versorgung am 24. Juni 2015 im Kursaal Bern}

Das Nationale Symposium 2015 des Schweizer Forum für Integrierte Versorgung fmc beleuchtet unter dem Titel «Mehr Patientensicht - mehr Integration" die Patientenperspektive als unentbehrliche Quelle, um die einzelnen Behandlungsschritte besser aufeinander abzustimmen. Persönlichkeiten aus dem Schweizer Gesundheitswesen liefern Impulse, wie sich die Patientenperspektive einsetzen lässt und mit welchen Fördermassnahmen die Integration und damit die Versorgungsqualität verbessert werden können. «Mehr Diskussion - mehr Nutzen» ist der zweite Fokus des Symposiums: In moderierten Tischgesprächen bringen die Teilnehmenden ihre Perspektive und Erfahrungen ein. Neu wird das Symposium in Bern mit Simultanübersetzung in deutscher und französischer Sprache durchgeführt. Weitere Informationen zum fmc-Symposium 2015 finden Sie auf ww.fmc.ch

Das Symposium 2015 wird vom Schweizerischen Institut für ärztliche Weiter- und Fortbildung (SIWF) mit 5 Credits anerkannt. 


\section{Literatur}

1 Delbanco TL. Quality of care through the patient's eyes. Brit Med J. 1996;313:832-3.

2 Barry JB. Shared Decision Making - The Pinnacle of PatientCentered Care. New Engl J Med. 2012;366: 780-1.

3 Noest S, Ludt S, Klingenberg A, Glassen K, Heiss F, Ose D, Rochon J, Bozorgmehr K, Eensing M, Szecseny J. Involving patients in detecting quality gaps in a fragmented healthcare system: development of a questionnaire for Patients' Experiences Across Health Care Sectors. Int J Qual Health Care. 2014;26:240-9.

4 NHS Department of Health. Liberating the NHS: No decision about me, without me. 2012. www.gov.uk/government/uploads/ system/uploads/attachment_data/file/216980/Liberatingthe-NHS-No-decision-about-me-without-me-Governmentresponse.pdf
5 Walker KO, Labat A, Choi J, Schmittdiel J, Stewart AL, Grumbach K. Patient perceptions of integrated care: confused by the term, clear on the concept. Int J Integr Care. 2013;Jan-Mar.

6 Juhnke C, Mühlbacher A. Patient-centeredness in integrated healthcare delivery systems - needs, expectations and priorities for organised healthcare systems. Int J Integr Care. 2013; Oct-Dec.

7 Prosser S. Being patient: Communication and its impact on innovation through the eyes of a patient (and former healthcare senior manager). Int J Healthcare Man. 2010;3:13-30. 